\title{
1006
}

\section{Bounds for Viscoelastic Properties of Heavy-oil Rocks} D. Makarynska* (Curtin University of Technology) \& B. Gurevich (Curtin
University of Technology/CSIRO)

\section{SUMMARY}

Heavy oils exhibit viscoelastic behaviour which is strongly frequency- and temperature- dependent. Due to the viscoelastic rheology of heavy oils the common elastic bounding methods such as Hashin-Shtrikman bounds are not rigorous for heavy-oil rocks. In this paper, we demonstrate that viscoelastic bounds of Milton and Berryman for the effective shear modulus of a two phase three-dimensional isotropic composite can be used as rigorous bounds for heavy-oil rocks. The viscoelastic bounds provide an effective tool for testing laboratory measurements and theoretical predictions for heavy-oil rocks. 


\section{Introduction}

Unlike common reservoir fluids (e.g., brine and light oil) heavy oils exhibit viscoelastic behaviour which is strongly frequency- and temperature- dependent. Heavy oil can act as a nearly elastic solid with a non-negligible shear modulus at relatively high frequencies and low temperatures, and as a Newtonian liquid at relatively low frequencies and high temperatures. The behaviour of heavy oil within a rock dominates the mechanical response of the rock to changes in temperature and frequency (Behura et al., 2007).

One result of viscoelastic rheology of heavy oils is that shear velocity in rocks in the seismic frequency band would significantly differ from that in the sonic logging-frequency range and the ultrasonic band. Therefore, the most common poroelastic theories of Gassmann (1951) and Biot (1956) can not be applied to heavy-oil rocks. The attempts to model elastic properties of heavy-oil rocks are very sparse containing a handful of papers where the results of modelling are compared with laboratory data (Leurer and Dvorkin, 2006; Gurevich et al., 2008; Das and Batzle, 2008; Makarynska et al., 2010).

Accurate laboratory data are important for establishing the right model. However, laboratory measurements of velocity dispersion are rather difficult and require development of special measurement techniques (Han et al. 2007). To date, few laboratory studies have been published in literature (Nur et al., 1984; Batzle et al., 2006; Behura et al., 2007; Han et al., 2008). While the measurements provide physical understanding of complex properties of heavy oil, their accuracy can be affected by different sources of errors such as poor quality of P- and especially S-wave signals, inadequate temperature and pore pressure control, difficulties in preserving integrity of a heavy oil sample, etc.

Rigorous bounding methods could provide a benchmark for testing laboratory measurements and results of modelling. They can also be used as an indicator as to whether the viscoelastic response of a given composite is extreme in the sense of being close to the edge of the bound. The best elastic bounds giving the narrowest range without specifying anything about the geometries of the constituents are Hashin-Shtrikman (HS) bounds (Mavko et al., 1998). These bounds are constrained by requirement of isotropy, and thus correspond to realistic geometrical structures of rocks. It is known that a correspondence principle between elasticity and viscoelasticity can be used to describe the linear response of viscoelastic composites at sufficiently low frequencies of oscillation. This means that HS bounds can be applied to solid/viscoelastic fluid mixtures. However in this case, the HS bounds become complex and are not longer rigorous as the notion of a value lying between two bounds is unidentified for complex numbers. HS bounds could provide rigorous realizable estimates only for the limiting cases of low temperature (elastic solid pore fill) and high temperature (Newtonian fluid pore fill). For the viscoelasticity problem, the set of possible effective moduli should fill a region in the complex plane and bounds should be represented as closed curves.

In this paper, we apply rigorous bounds (Milton and Berryman, 1997) especially designed for two-phase viscoelastic composites to the complex shear modulus measured on Uvalde heavy-oil rock sample (Behura et al., 2007). We also apply the bounds to the results of modelling of elastic properties by self-consistent effective medium theory known as Coherent Potential Approximation or CPA (Berryman, 1980). We restrict our analysis to shear properties only because they are more sensitive to temperature changes in reservoirs than bulk properties.

\section{Rigorous viscoelastic bounds}

Milton and Berryman (1997) extended HS bounds for the effective shear-modulus to viscoelasticity using variational principles (for derivation details and the algorithm see Milton and Berryman, 1997; Gibiansky and Milton, 1993). They considered the dynamic response of statistically isotropic three dimensional composites with two viscoelastic isotropic phases mixed in fixed proportions in the quasi-static regime or at frequencies where the wavelength of a passing wave is much larger than the inhomogeneities (inclusions). The bounding regions in a complex plane are composed by arcs of circles containing four points related to the bulk and shear moduli of the constituents. The resulting bounds form a lens shaped region obtained by taking the intersection of all such arcs. 
Similarly to HS bounds, the viscoelastic bounds are independent of the microstructure of a rock and the only input parameters are needed to construct the bounding curves are the constituent bulk and shear moduli and the volume fractions of the constituents. The method is relatively easy to apply in practice due to simplified parameterization used in the derivation. On the other hand, these simplifications could make the resulting bounds less tight than if extracted from the more general theory. Importantly, the points which correspond to HS bounds on the shear modulus always lie inside or on the boundary of the bounding region. In the limiting case, when all the constituent moduli are real the viscoelastic bounds reduce precisely to HS bounds.

\section{Uvalde heavy-oil rock}

In our modelling, we use laboratory measurements of temperature- and frequency-dependent shear modulus carried out on Uvalde heavy-oil rock samples from Texas, USA as reported in Behura et al. (2007). These measurements represent important conditions encountered during in situ steam flooding of heavy oil reservoirs.

The Uvalde rock is a carbonate saturated with extremely viscous heavy oil with API density of $5(-1.12 \mathrm{gm} / \mathrm{cc})$. It has a porosity of approximately $25 \%$, and permeability of $550 \mathrm{mD}$. A shear rheometer was used to measure the complex shear modulus of the rock at temperatures ranging from $30^{\circ} \mathrm{C}$ to $350^{\circ} \mathrm{C}$ and frequencies ranging from 0.01 to $80 \mathrm{~Hz}$.

The extracted heavy oil was measured at temperatures ranging from $30^{\circ} \mathrm{C}$ to $250^{\circ} \mathrm{C}$ and the same frequency range. All measurements were made in the linear viscoelastic regime. As it was pointed out by Behura et al. (2007), strain amplitude lying within this linear region was selected for conducting all temperature-frequency measurements. It is important to note that the strain limit for linear behaviour varies from one rock or material to another. The same strains $\left(6 \times 10^{-5}-8 \times 10^{-5}\right)$ were used for testing heavy-oil rocks and the extracted oil. Note that the used strains are more than one order larger than the strains encountered in exploration seismology. This might result in laboratory measured properties different from seismic ones.

\section{CPA modelling}

Effective medium theories can be used to provide specific estimated values for complex shear modulus. Self-consistent effective medium theory or CPA (Berryman, 1980) is known to have definite microstructures associated with it (Milton, 1985) and therefore it is (at least in principle) realizable.

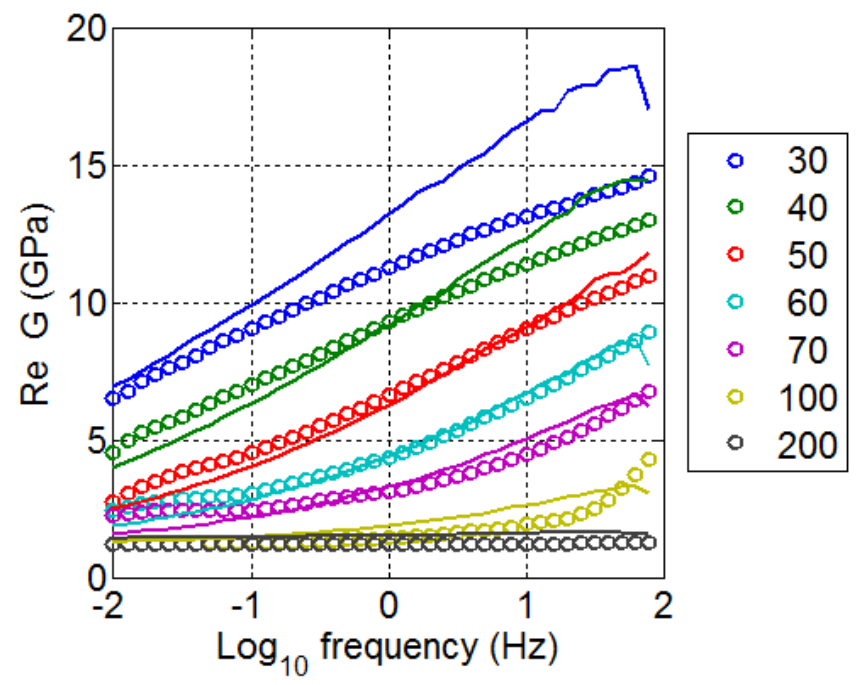

Figure 1 Comparison of frequency- and temperature-dependent shear modulus $G$ (real part) predicted by CPA (symbols) with laboratory measurements carried out on Uvalde heavy-oil rock sample (lines). 
That means that the predicted values will always lie inside the rigorous bounds, although those microstructures may not always be realistic representations of the microstructure of interest in a particular application.

Makarynska et al. (2010) used self-consistent effective medium theory known as CPA (Berryman, 1980) as an alternative fluid substitution technique to predict shear properties of Uvalde heavy-oil rock. The results of modelling are compared with measurements in Figure 1. We can observe a very good agreement between the CPA predictions (symbols) and the laboratory data (lines) with the only deviation larger than the measurement error (approximately $\pm 2.09 \mathrm{GPa}$, see Behura et al., 2007) observed at high frequencies for $30^{\circ} \mathrm{C}$. In order to understand this discrepancy, we use the viscoelastic bounds for testing the results of modelling and laboratory data.

\section{Application of viscoelastic bounds}

We calculate the bounds for the range of frequencies and temperatures. Figure 2 shows the bounds calculated for the shear modulus $G$ and inverse quality factor $1 / Q$ of Uvalde heavy-oil rock: (a) at the temperature and frequency of the largest mismatch between CPA estimates and laboratory data when the oil inside the rock is close to solid state and (b) when the predictions and laboratory data are in a good agreement and the oil is in transition between solid and liquid state.

(a)

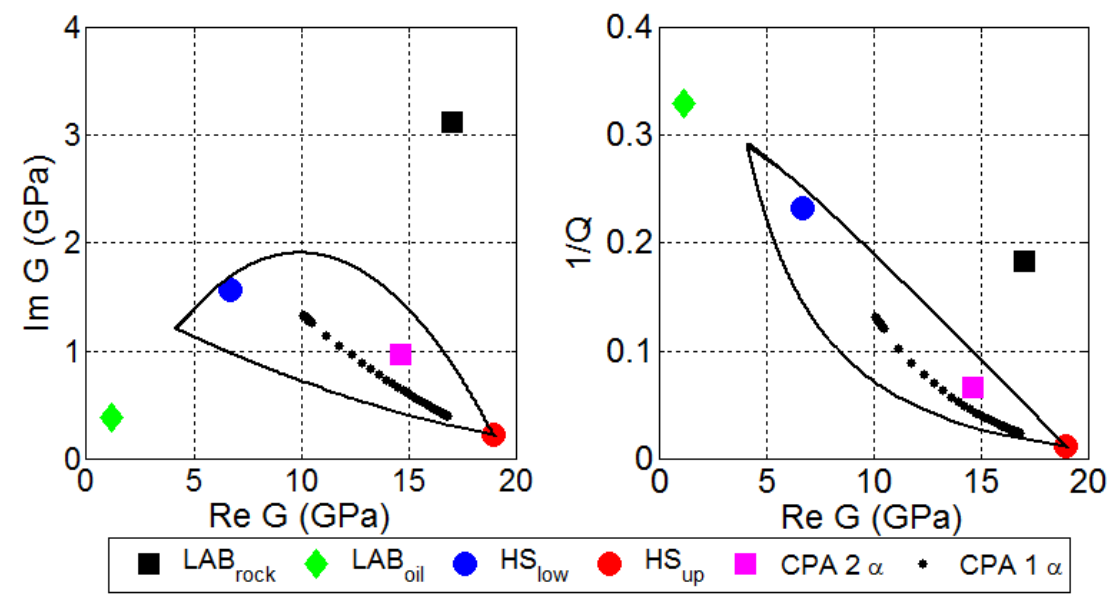

(b)

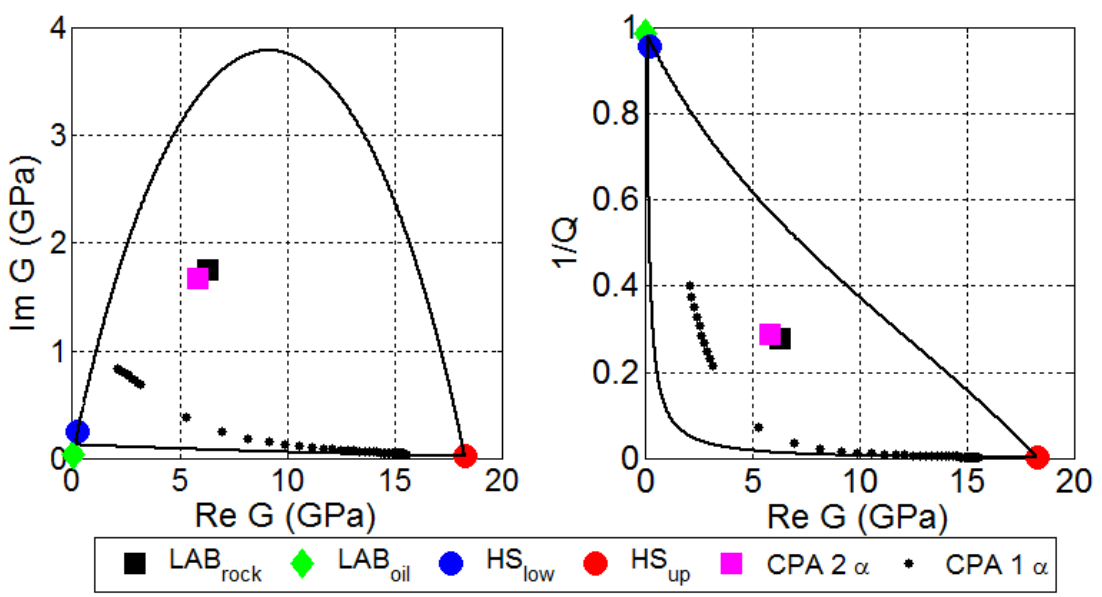

Figure 2 Viscoelastic bounds (black lines) for the effective shear modulus $G$ (left panels) and inverse quality factor $1 / Q$ (right panels) of Uvalde heavy-oil rock: (a) $T=30^{\circ} \mathrm{C}$ and $f=79 \mathrm{~Hz}$, (b) $T=70^{\circ} \mathrm{C}$ and $f=40 \mathrm{~Hz}$. CPA predictions of $G$ (magenta squares) are compared to laboratory data (black squares). Also shown: CPA estimates of $G$ for a range of aspect ratios (black dots), HS lower (blue circles) and upper (red circles) bounds, and measured $G$ of heavy oil (green diamonds). 
As input parameters we use properties of calcite ( $K=60 \mathrm{GPa}, G=30 \mathrm{GPa}$ ) and properties of heavy oil with the laboratory measured shear modulus ( $K=3 \mathrm{GPa}, G=1.1+0.4 \mathrm{i} \mathrm{GPa}$ ). In Figure 2a, we can see that CPA estimates lie within the bounds, while measured $G$ of Uvalde heavy-oil rock is outside. This suggests that the mismatch between the predictions and laboratory data is caused by measurement errors. Note that the bounds are quite narrow for the imaginary part of $G$ (loss modulus) responsible for the energy dissipation. This also applies to the inverse quality factor. This is quite physical as the heavy oil in these temperature and frequency conditions is nearly a solid.

As a test, we also plot CPA estimates for the aspect ratios ranging from 0 to 1 (black dots), which represent different pore geometries. As expected, all the CPA estimates lie within the bounds. When temperature increases and frequency becomes lower, the bounding curves become wider (Figure 2b). To construct the bounds for this case, we use the measured oil shear modulus $G=0.03+0.03 \mathrm{i} \mathrm{GPa}$ and the same other parameters as in the previous example. Figure $2 \mathrm{~b}$ shows that CPA estimates and laboratory data agree very well and are contained within the bounds.

\section{Conclusions}

We have demonstrated that viscoelastic bounds of Milton and Berryman (1997) for the effective shear modulus of a two phase three-dimensional isotropic composite can be used as rigorous bounds for heavy-oil rocks. The viscoelastic bounds provide an effective tool for testing laboratory measurements and theoretical predictions for heavy-oil rocks.

It is important to note that these bounds are quasi-static and are designed to work at frequencies where the wavelength is much larger than the inhomogeneities. Simplified parameterization used in the derivation can result in wider bounds. Alternatively, more restrictive bounds developed for a twodimensional composite (Gibiansky et. al, 1999) can also be applied to rocks saturated with viscoelastic fluids.

\section{References}

Batzle, M., R. Hofmann, and D.-H. Han, 2006. Heavy oils-seismic properties. The leading Edge, 25, 750-757.

Behura, J., M. Batzle, R. Hofmann, and J. Dorgan, 2007, Heavy oils: Their shear story: Geophysics, 72, no. 5, E175-E183.

Berryman, J. G., 1980, Long-wavelength propagation in composite elastic media II. Ellipsoidal inclusions: Journal of the Acoustical Society of America, 68, 1980-1831.

Biot, M. A, 1956, Theory of propagation of elastic waves in a fluid saturated porous solid. II. Higher frequency range: Journal of the Acoustical Society of America, 28, 179-191.

Das A., and M. Batzle, 2008, Modeling studies of heavy oil-in between solid and fluid properties: The Leading Edge, 27, 1116-1123.

Gassmann, F., 1951, Uber die Elastizitat poroser Medien (Elasticity of porous media): Vierteljahrsschrift der Naturforschenden Gesselschaft in Zurich, 96, 1-23.

Gibiansky, L. V., and G. W. Milton, 1993, On the effective viscoelastic moduli of two-phase media. I. Rigorous bounds on the complex bulk modulus: Proceedings of the Royal Society of London A, 440, 163-188.

Gibiansky, L. V., G. W. Milton, and J. G. Berryman, 1999, On the effective viscoelastic moduli of two-phase media. III. Rigorous bounds on the complex shear modulus in two dimensions: Proceedings of the Royal Society of London A, 455, 2117-2149.

Gurevich, B., K. Osypov, R. Ciz, and D. Makarynska, 2008, Modeling elastic wave velocities and attenuation in rocks saturated with heavy oil: Geophysics, 73, no. 4, E115-E122.

Han, D.-H., H.-Z. Zhao, and Q. Yao, 2007, Velocity of heavy oil sand: 77th Annual International Meeting, SEG, Expanded Abstracts, 1619-1623.

Han, D.-H., J. Liu, and M. Batzle, 2008, Seismic properties of heavy oils-measured data: The Leading Edge, 27, 1108-1115. Leurer, K. C., and J. Dvorkin, 2006, Viscoelasticity of precompacted unconsolidated sand with viscous cement: Geophysics, 71, T31-T40.

Makarynska, D., B. Gurevich, J. Behura, and M. Batzle, 2010, Fluid substitution in rocks saturated with viscoelastic fluids: Geophysics, accepted for publication.

Mavko, G., T. Mukerji, and J. Dvorkin, 1998, The Rock Physics Handbook: Tools for seismic analysis in porous media: Cambridge University Press.

Milton, G. W., 1985, The coherent potential approximation is a realizable effective medium scheme: Communications in Mathematical Physics, 99, 463-500.

Milton, G. W., and J. G. Berryman, 1997, On the effective viscoelastic moduli of two-phase media. II. Rigorous bounds on the complex shear modulus in three dimensions: Proceedings of the Royal Society of London A, 453, 1849-1880.

Nur, A., C. Tosaya, and D. V. Thanh, 1984, Seismic monitoring of thermal enhanced oil recovery processes: 54th Annual International Meeting, SEG, Expanded Abstracts, 118-121. 